\title{
Impacto do Exercício Físico Isolado e Combinado com Dieta Sobre os Níveis Séricos de HDL, LDL, Colesterol Total e Triglicerídeos
}

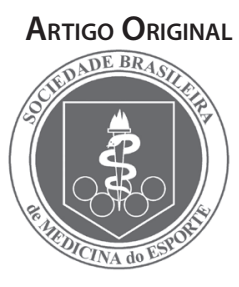

\author{
Impact Of Isolated And Combined With Diet Physical Exercise On The \\ Hdl, Ldl, Total Cholesterol And Triglycerides Plasma Levels
}

\begin{abstract}
Sanmira Fagherazzi
Raquel da Luz Dias²

Fernanda Bortolon

1. Centro Integrado de Medicina do Exercício (CIME) do Hospital Mãe de Deus (HMD) - Porto Alegre/RS.

2. Faculdade de Nutrição da Pontifícia Universidade Católica do Rio Grande do Sul (PUCRS) - Porto Alegre/RS
\end{abstract}

\section{Endereço para correspondência: Sanmira Fagherazzi Av. Capivari, 550, Bairro Cristal 90810070 - Porto Alegre/RS \\ E-mail: sanmira@hotmail.com}

Submetido em 03/03/2006 Versão final recebida em 04/02/2007 Aceito em 30/03/2007

\begin{abstract}
RESUMO
Adequados hábitos alimentares e a prática de exercícios físicos exercem efeito benéfico sobre as dislipidemias. Se associados, podem ainda otimizar as mudanças do perfil lipoprotéico plasmático, sendo, além disso, intervenções de custo moderado quando comparados com tratamentos medicamentosos e dependentes de alta tecnologia. Este estudo tem por objetivo avaliar o impacto do exercício físico isolado e combinado com dieta sobre o perfil lipídico em indivíduos com sobrepeso/obesos. O presente trabalho é do tipo retrospectivo analítico observacional. Nele foi analisada a evolução do perfil lipídico e do peso, por período entre três e seis meses, de 30 indivíduos, divididos em dois grupos: grupo exercício (prática de exercício físico) e grupo dieta (prática de exercício físico associada à intervenção nutricional). Foram encontradas reduções estatisticamente significativas no CT $(-14,4 \mathrm{mg} / \mathrm{dl} ; \mathrm{P}=0,022)$ e no LDL-c $(-20,9 \mathrm{mg} / \mathrm{dl}$; $P=0,013)$ para os componentes do grupo exercício. Tal redução também ocorreu em relação à razão CT/ HDL-c $(-0,9 ; P=0,005)$ para os componentes do grupo dieta. Foi observada elevação dos níveis de HDL-c, apenas no grupo dieta $(+4,2 \mathrm{mg} / \mathrm{dl})$. Nesse mesmo grupo verificou-se diminuição no $\mathrm{CT}(-8 \mathrm{mg} / \mathrm{dl})$ e no LDL-c $(-9,8 \mathrm{mg} / \mathrm{dl})$, bem como redução de peso $(-2,6 \mathrm{~kg})$, no entanto, tais resultados não foram estatisticamente significativos. Quanto aos níveis de TG, não foi verificada evolução positiva em ambos os grupos. Concluiu-se que o efeito isolado do exercício físico foi mais evidente em relação às variáveis CT e LDL-c. Os TG não sofreram modificações positivas com a prática exclusiva de exercícios físicos ou com sua associação à dieta. Para as variáveis HDL-c e peso, a combinação da dieta com o exercício físico apresentou maiores benefícios.
\end{abstract}

Palavras-chave: nutrição, atividade física, dislipidemia, obesidade, doenças cardiovasculares.

\section{ABSTRACT}

Adequate eating habits and physical exercise have a beneficial effect on dislipidemies. When associated, they might even optimize changes to the plasmatic lipoproteic profile, apart of which they are moderatecost interventions if compared to drug-based and high-tech depending treatments. The present study aims at assessing the impact of physical exercise as isolated and combined with a diet on the lipidic profile of overweight/obese individuals. Tn observational analytical retrospective study has looked into the evolution of the lipidic profile and weight over a period of 3 to 6 months of 30 individuals divided in two groups: the exercise group (physical exercise practice) and the diet group (physical exercise associated with a nutritional intervention). Significant statistical reductions were found in the CT $(-14.4 \mathrm{mg} / \mathrm{dl} ; \mathrm{P}=0,022)$ and in the LDL-C $(-20.9 \mathrm{mg} / \mathrm{dl} ; \mathrm{P}=0,013)$ for the components in the exercise group. Such reduction has also occurred regarding the $C T / H D L-c(-0,9 ; P=0,005)$ ratio for the components of the diet group. The increase in the HDL-c levels was observed only in the diet group $(+4.2 \mathrm{mg} / \mathrm{dl})$. In this same group a decrease in the CT $(-8 \mathrm{mg} / \mathrm{dl})$ and in the LDL-c $(-9,8 \mathrm{mg} / \mathrm{dl})$ was observed as well as a weight reduction $(-2.6 \mathrm{Kg})$, however, such results have not been statistically significant. Regarding the TG levels, there was no evidence for a positive evolution in either group. As a conclusion, the isolated effect of physical exercise was more evident concerning the variables CT and LDL-c. The TG did not undergo positive modifications upon the exclusive practice of physical exercise or with their association with the diet. As for variables HDL-c and weight, the combination of diet and physical exercise has proven to bring enhanced benefits.

Keywords: nutrition, physical activity, dyslipidemia, obesity, cardiovascular diseases. 


\section{INTRODUÇÃO}

A relevância das dislipidemias como problema de saúde pública está na sua relação com as doenças cardiovasculares (DCV)(1), estando classificadas entre os mais importantes fatores de risco para doença cardiovascular aterosclerótica, juntamente com a hipertensão arterial, a obesidade e o diabetes mellitus ${ }^{(2)}$.

As DCV constituem uma importante causa de morte nos países desenvolvidos e também naqueles em desenvolvimento, onde o seu crescimento significativo alerta para o profundo impacto nas classes menos favorecidas e para a necessidade de intervenções eficazes de baixo custo e caráter preventivo ${ }^{(3)}$. Segundo dados do Atlas de doenças cardíacas e derrames da Organização Mundial da Saúde $(\mathrm{OMS})^{(4)}$, a cada ano morrem 17 milhões de pessoas em todo o mundo devido às doenças cardiovasculares. No Brasil, em 2002, tais doenças foram responsáveis por cerca de 268.773 mortes $^{(4)}$. Atualmente, as doenças cardiovasculares representam 9,5\% das internações, as quais equivalem a 17\% dos gastos do SUS - Sistema Único de Saúde, e, segundo o Ministério da Saúde, são responsáveis por 24\% das aposentadorias por invalidez ${ }^{(5)}$.

As dislipidemias podem ser definidas como distúrbios do metabolismo lipídico, com repercussões sobre os níveis de lipoproteínas na circulação sanguínea, bem como sobre as concentrações de seus diferentes componentes ${ }^{(6)}$. A lipoproteína de baixa densidade (LDL-c) é o maior carreador de colesterol para as células ${ }^{(5)}$ e está associado ao início e à aceleração do processo aterosclerótico(7). Já as lipoproteínas de alta densidade (HDL-c), são de extrema importância, pois participam do transporte reverso do colesterol, sendo consideradas antiaterogênicas $s^{(5)}$.

Ainda que a genética, o sexo e a idade sejam de grande importância para o desenvolvimento das dislipidemias, a mudança de hábitos alimentares e a prática de atividade física são modificações no estilo de vida que podem melhorar de forma significativa essas doenças ${ }^{(3)}$. Se associadas, essas práticas podem ainda otimizar as mudanças do perfil lipoprotéico plasmático (2), sendo, além disso, intervenções de baixo custo, se comparadas com tratamentos medicamentosos ${ }^{(3)}$.

Já foi constatado que hábitos alimentares inadequados são a principal causa do surgimento de dislipidemias ${ }^{(8)}$. A gordura saturada é a principal causa alimentar da elevação do colesterol plasmático(9). Os ácidos graxos saturados (AGS) estão relacionados com o aumento do colesterol total e do LDL-c, bem como com a elevação dos triglicerídeos $(\mathrm{TG})^{(10)}$. As gorduras transisoméricas também provocam elevação da colesterolemia, com a desvantagem de reduzir o HDL-C(2). O colesterol dietético está diretamente relacionado com a elevação do LDL-( ${ }^{(11)}$, porém, possui menor efeito sobre a colesterolemia, quando comparado com as gorduras saturadas ${ }^{(3)}$.

Por outro lado, às gorduras monoinsaturadas têm sido relacionadas com melhoras nos níveis de TG, diminuição do colesterol total e do LDL-c, aumentando também os níveis de HDL-c no plasma, característica importante no contexto da redução dos riscos cardiovasculares ${ }^{(12)}$. As gorduras poliinsaturadas (ácidos graxos ômega 3 e ômega 6) também exercem efeito positivo sobre o colesterol total, LDL-c e TG séricos ${ }^{(2,13)}$. Porém, os ácidos graxos ômega 6 , embora considerados não prejudiciaisis), induzem maior oxidação lipídica ${ }^{(2)}$ e há indícios de que possam reduzir as concentrações de $\mathrm{HDL}-\mathrm{C}^{(14)}$. A American Heart Association indica, para prevenção primária da doença aterosclerótica coronariana, consumo diário entre $8 \%$ e 10\% de ácidos graxos saturados; até $10 \%$ de poliinsaturados; até 15\% de monoinsaturados e até 300mg de colesterol. Para prevenção secundária, preconizam-se até $7 \%$ de ácidos graxos saturados e restrição de até $200 \mathrm{mg}$ de colesterol por dia ${ }^{(15)}$.
Além dos lipídios, outros fatores dietéticos afetam as concentrações plasmáticas de colesterol e lipoproteínas; é o caso das fibras solúveis, dos antioxidantes, do café e do álcool, além de alguns componentes funcionais ${ }^{(16)}$.

O combate às dislipidemias através do exercício físico vem sendo alvo de inúmeros estudos e debates científicos em todo o mundo. Atualmente, essa prática está sendo recomendada como parte integrante do tratamento dessas doenças ${ }^{(17-18)}$. Um dos maiores benefícios da atividade física regular é a melhora do perfil lipídico em longo prazo(19). O tipo de exercício que mais atua no metabolismo de lipoproteínas é o aeróbio ${ }^{(20)}$, porém, exercícios de força e flexibilidade também são recomendados ${ }^{(2)}$.

Bons hábitos alimentares e a prática de atividade física, além dos benefícios já relatados, favorecem ainda, o equilíbrio entre a ingestão alimentar e o gasto energético diário, o que previne a obesidade, a qual, por sua vez, representa um forte determinante para o desenvolvimento e a manutenção de quadros típicos de dislipidemias ${ }^{(21)}$.

Embora vários estudos investiguem o efeito de nutrientes isolados ou apenas da prática de exercícios físicos sobre o perfil lipoprotéico plasmático, a abordagem simultânea da nutrição e atividade física é importante, pois engloba grande parte das mudanças de estilo de vida necessárias ao controle das dislipidemias e, conseqüentemente, a prevenção de $\mathrm{DCV}^{(3)}$. Além disso, quanto ao impacto isolado do exercício sobre o HDL-c, LDL-c e TG, os estudos são ainda insuficientes para uma posição definitiva em relação a subgrupos, como o caso de obesos e idosos ${ }^{(2)}$, que representam populações extremamente suscetíveis a desenvolver esse tipo de alterações.

Assim, a intenção desta pesquisa é avaliar o impacto do exercício físico isolado e combinado com dieta sobre o perfil lipídico de indivíduos com sobrepeso/obesos, a fim de obter-se, através dos achados, embasamento científico para melhor conduta profilática e terapêutica.

\section{METODOLOGIA}

Este estudo, cujo delineamento é do tipo retrospectivo analítico observacional, constou de 30 indivíduos, fisicamente ativos, portadores de doenças cardiovasculares, e/ ou portadores de fatores de risco desencadeantes dessas doenças, encaminhados por profissionais da área da saúde ao Centro Integrado de Medicina do Exercício-CIME do Hospital Mãe de Deus, em Porto Alegre.

Os participantes foram divididos em dois grupos de acordo com o período no qual freqüentaram o CIME: GE (grupo exercício), quando o Serviço de Nutrição ainda não havia sido implantado, e GD (grupo exercício + dieta), após a sua implementação.

O GE foi composto por 15 indivíduos que praticaram atividade física regularmente em um prazo de três a seis meses, no período de janeiro de 2000 a dezembro de 2003, quando não usufruíam de supervisão nutricional.

O GD também foi composto por 15 indivíduos que praticaram atividade física regularmente, em um prazo de três a seis meses, no período de agosto de 2004 a julho de 2005. Diferentemente do GE, os componentes desse grupo receberam supervisão nutricional durante o período em questão.

O programa de exercícios físicos para os dois grupos foi realizado com freqüência de três vezes por semana, intensidade de 70 a 80\% da freqüência cardíaca e $\mathrm{VO}_{2}$ entre 60 e $85 \%$. O treino tinha duração de uma hora, das quais: cinco minutos de aquecimento, 25 minutos de atividade aeróbica, 20 minutos de exercício de resistência dos grandes grupos musculares (duas séries de 12 repetições com 40 a 60\% de 1RM - uma repetição máxima), e 10 minutos de alongamento. 
Quanto ao aconselhamento dietético, realizado exclusivamente no GD, os pacientes eram submetidos à avaliação nutricional e recebiam plano alimentar quando iniciavam o programa, não sendo obrigatório seu retorno para reconsulta. A dieta foi calculada através do software de Avaliação Nutricional dietWin Clínico versão 1999-2002, e consistiu basicamente, em prescrição de dieta hipocalórica - visando perda ponderal de 1,5 a 2,5kg/mês - com reduzido valor de gordura saturada, gordura trans, colesterol e adequados níveis de gordura poli e monoinsaturadas, proteínas e fibras. A dieta foi individualizada, obedecendo, quando possível, às preferências e hábitos alimentares relatados e elaborada de acordo com as recomendações da American Heart Association ${ }^{(15)}$.

Os critérios de inclusão para o GE foram: ter idade $\geq 45$ anos, apresentar índice de massa corporal (IMC) correspondente a sobrepeso $\left(25-29,9 \mathrm{~kg} / \mathrm{m}^{2}\right)$ ou obesidade $\left(\geq 30 \mathrm{~kg} / \mathrm{m}^{2}\right)^{(22)}$, e praticar atividade física regularmente por, no mínimo, três meses. Os critérios de inclusão para o GD foram os mesmo do GE, acrescentando-se apenas a necessidade de os participantes terem recebido supervisão nutricional durante o período analisado.

Por sua vez, os critérios de exclusão para o GE foram definidos como: utilizar medicação hipolipemiante durante o período em estudo, apresentar diabetes mellitus tipo I ou II, utilizar álcool regularmente, ser fumante, possuir histórico de dislipidemia familiar, estar sob tratamento dietoterápico durante o período em estudo, ou não se encaixar em algum dos critérios de inclusão. Já para o GD, exceto a exigência de não estar sob tratamento dietoterápico, todos os critérios de exclusão foram os mesmos do GE.

Foram coletados dados de prontuário referentes aos níveis séricos de colesterol total (CT), LDL-C, HDL-c e TG, bem como peso e altura, para análise do perfil lipídico e possível correlação com atividade física e/ou dieta. Os dados de ambos os grupos são referentes a dois momentos distintos: o primeiro, quando da entrada do indivíduo no CIME, e o segundo, três meses após essa data, no caso do peso, e de três a seis meses após essa data, no caso do perfil lipídico. Todos os dados coletados são rotinas de serviço do CIME, fazendo parte do banco de dados existente no Serviço, não havendo, portanto, contato direto com os participantes da pesquisa para a coleta dos mesmos.

A pesagem e a verificação da altura foram realizadas em balança digital com antropômetro da marca Filizola, modelo PL 180, com capacidade para $180 \mathrm{~kg}$ e precisão de $100 \mathrm{~g}$.

Os exames bioquímicos foram realizados por laboratórios particulares e a coleta de sangue feita após 12 horas de jejum. Os níveis de referência dos lipídios séricos foram definidos, de acordo com as diretrizes da Sociedade Brasileira de Cardiologia(2), sendo também calculados os índices de risco de Castelli I e || (razão CT/HDL-c e razão LDL-C/HDL-c, respectivamente) ${ }^{(23)}$.

Os dados foram tabulados no programa SPSS (Statistical Package for the Social Sciences), versão 10.0 for Windows. Foi realizada estatística descritiva, com mediana e percentis 25-75, ou média e desvio-padrão para as variáveis quantitativas, e freqüências absolutas e relativas para as variáveis qualitativas. Para comparar as variáveis antes e após intervenção, foi utilizado o teste $t$ de Student para amostras pareadas ou o teste de Wilcoxon. Para as comparações entre grupos, foi utilizado o teste do qui-quadrado de Pearson para as variáveis qualitativas, e os testes $t$ de Student para amostras independentes, ou Mann-Whitney para as variáveis quantitativas. O nível de significância adotado foi de $5 \%$ (24).

Foi assinado termo de compromisso para utilização de dados, no qual as autoras desta pesquisa se comprometeram a manter o sigilo dos dados coletados do banco de dados do Serviço, bem como a utilização destes exclusivamente com finalidade científica. O presente trabalho obteve aprovação da comissão científica e do comitê de ética da Pontifícia Universidade Católica do Rio Grande do Sul (PUCRS) e do Hospital Mãe de Deus (HMD).

\section{RESULTADOS}

Participaram desta pesquisa 30 indivíduos. A freqüência dos participantes por sexo foi de 15 do feminino e 15 do masculino, sendo no GE, 10 homens $(66,7 \%)$ e cinco mulheres (33,3\%), e no GD, 10 mulheres $(66,7 \%)$ e cinco homens (33,3\%). A média de idade ( \pm DP) foi de $62,2 \pm$ 20 anos, predominando no GE idade $\geq 60$ anos (80\%) e no GD, entre 45 e 59 anos (53,3\%)

De acordo com o IMC, os participantes do GE apresentaram, predominantemente, sobrepeso (86,7\%), enquanto no GD, o IMC indicativo de obesidade foi o mais encontrado (60\%). A média de peso ( \pm DP) foi de 78,9 $\pm 18,3 \mathrm{~kg}$ e $84,7 \pm 15,4 \mathrm{~kg}$, para o GE e GD, respectivamente.

Os valores médios/mediano de colesterol total (CT) e suas frações estão apresentados na tabela 1, porém, não foram encontradas diferenças estatisticamente significativas para as variáveis de lipídios séricos entre os grupos.

Ainda na tabela 1, nota-se que os dois grupos apresentaram valores médios/mediano de CT, LDL-C, HDL-c e TG, dentro do recomendado pela Sociedade Brasileira de Cardiologia (2). Para os índices de risco de Castelli I e II, em ambos os sexos, a média também permaneceu dentro dos valores aceitáveis para os dois grupos ${ }^{(23)}$.

Tabela 1. Caracterização da amostra no momento inicial da pesquisa, conforme o grupo

\begin{tabular}{|c|c|c|c|}
\hline \multirow[b]{2}{*}{ Características } & \multicolumn{2}{|c|}{ Grupo } & \multirow[b]{2}{*}{$\mathbf{P}$} \\
\hline & $\begin{array}{c}\text { Exercício } \\
(n=15)\end{array}$ & $\begin{array}{l}\text { Exercício + dieta } \\
\quad(n=15)\end{array}$ & \\
\hline \multicolumn{4}{|l|}{ Sexo $-n(\%)$} \\
\hline Masculino & $10(66,7)$ & $5(33,3)$ & $0,144^{*}$ \\
\hline Feminino & $5(33,3)$ & $10(66,7)$ & \\
\hline Idade - Média (DP) & $66,5(10,7)$ & $58(9,2)$ & $0,027^{* *}$ \\
\hline \multicolumn{4}{|l|}{ Faixa etária - n(\%) } \\
\hline$<60$ anos & $3(20)$ & $8(53,3)$ & $0,130^{*}$ \\
\hline$\geq 60$ anos & $12(80)$ & $7(46,7)$ & \\
\hline Altura - Média (DP) & $1,68(0,09)$ & $1,61(0,06)$ & $0,029^{* *}$ \\
\hline Peso - Média (DP) & $78,9(18,3)$ & $84,7(15,4)$ & $0,356^{* *}$ \\
\hline IMC - Média (DP) & $27,9(4,5)$ & $32,8(6,3)$ & $0,020^{* *}$ \\
\hline $\begin{array}{l}\text { Colesterol total - } \\
\text { Média (DP) }\end{array}$ & $209,4(30,6)$ & $191,5(34,3)$ & $0,142^{* *}$ \\
\hline LDL - Média (DP) & $139,5(39,1)$ & $122(33,8)$ & $0,201 * *$ \\
\hline HDL - Média (DP) & $46,7(13,2)$ & $43,3(9,8)$ & $0,420^{* *}$ \\
\hline $\begin{array}{l}\text { Triglicerídeos - Mediana } \\
\text { (P25 - P75) }\end{array}$ & $124(81-174)$ & $113(101-174)$ & $0,870^{* * *}$ \\
\hline Razão CT/HDL - Média (DP) & $4,7(1,3)$ & $4,6(1,2)$ & $0,759^{* *}$ \\
\hline Razão LDL/HDL - Média (DP) & $3(1,2)$ & $2,9(0,9)$ & $0,276^{* *}$ \\
\hline
\end{tabular}

DP=desvio padrão; P25=percentil25; P75=percentil 75

* Valor obtido pelo teste qui-quadrado de Pearson

** Valor obtido pelo teste-t Student

*** Valor obtido pelo teste de Mann-Whitney 
Tabela 2. Comparação entre dados antropométricos e perfil lipídico, antes e após intervenção, conforme o grupo

\begin{tabular}{l|c|c|c|c|c|c|}
\hline \multirow{2}{*}{ Características } & \multicolumn{2}{|c}{ Grupo } & \multicolumn{2}{c}{ Exercício + dieta } \\
(n=15)
\end{tabular}

$\mathrm{DP}=$ desvio padrão; $\mathrm{P} 25=$ percentil25; $\mathrm{P} 75=$ percentil 75

* Valor obtido pelo teste t de Student para amostras pareadas

** Valor obtido pelo teste de Wilcoxon

Comparando-se o peso inicial e final, observou-se decréscimo no grupo que sofreu intervenção nutricional, o qual obteve perda média de 2,6kg (tabela 2). No entanto, tal mudança não foi estatisticamente significativa $(P=0,218)$. O grupo que apenas praticou exercícios físicos permaneceu com média de peso praticamente inalterada, havendo apenas uma pequena elevação de $0,2 \mathrm{~kg}(P=0,745)$.

A redução de peso foi maior nas mulheres. Entre os participantes que obtiveram redução do peso inicial, 75\% eram do sexo feminino. Em relação à faixa etária, os indivíduos com idade inferior a 60 anos apresentaram pequena vantagem se comparados com os com mais de 60 anos, 45,4 e 31,6\%, respectivamente.

A análise estatística demonstrou não haver diferença significativa em relação à classificação do IMC em ambos os grupos ao final do programa; o GD obteve melhor resposta, apresentando redução do IMC em mais da metade dos componentes desse grupo (53\%).

Notou-se associação entre o índice de massa corporal e a relação CT/HDL-c. Esta associação foi avaliada através do coeficiente de correlação de Pearson, onde obteve-se uma relação positiva estatisticamente significativa $(r=0,566 ; P=0,028)$, como pode ser visualizado na figura 1. Não foram encontradas outras correlações entre parâmetros antropométricos e lipídios/lipoproteínas.

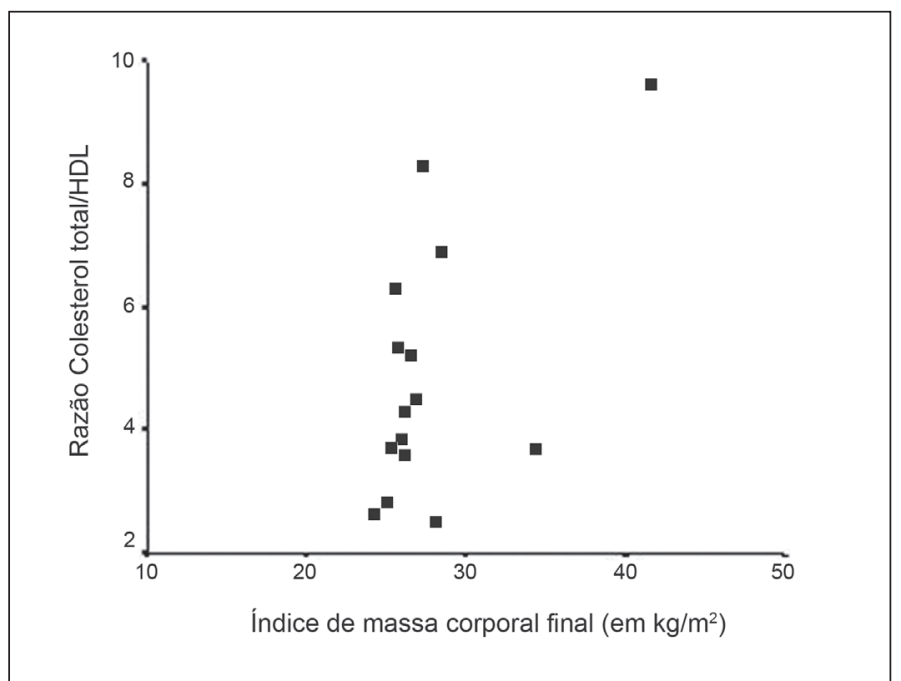

Figura 1. Relação entre Índice de Massa Corporal e razão CT/HDL, em praticantes de exercício físico
Quanto às análises do perfil lipídico, observou-se redução com significância estatística no $C T(P=0,022)$ e no $L D L-C(P=0,013)$ somente no GE. A figura 2 compara os valores médios iniciais e finais desses componentes lipídicos, relacionados ao grupo exercício. Em relação ao GD, não foi encontrada redução significante estatisticamente, porém, $60 \%$ dos participantes deste grupo obtiveram diminuição do CT e $66,7 \%$ reduziram os níveis de LDL-c. Contudo, a média de redução (-8mg/dl para o CT e -9,8g/dl para o LDL-c), foi inferior à alcançada pelo GE (-14,8mg/dl para o CT e -20,9mg/dl para o LDL-c). Tais aspectos podem ser observados na tabela 2.

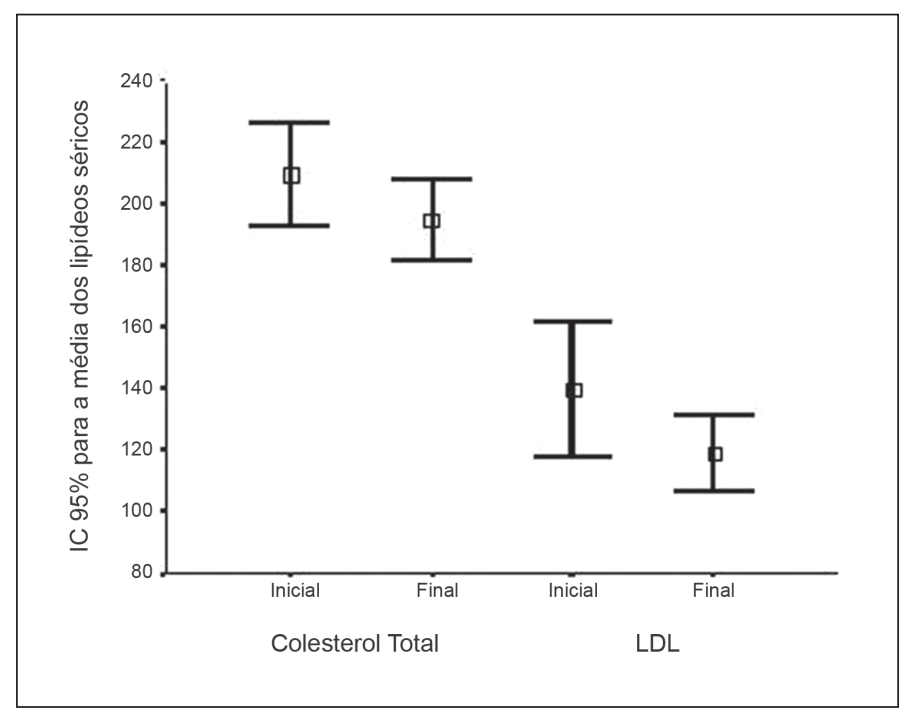

Figura 2. Comparação dos valores médios de colesterol total e LDL antes e após intervenção, no grupo exercício

O nível médio de HDL-c foi elevado apenas no grupo que recebeu intervenção nutricional $(+4,2 \mathrm{mg} / \mathrm{dl})$, apesar de esta mudança não ser estatisticamente significativa $(P=0,064)$. Não houve aumento no grupo que praticou exclusivamente exercícios físicos.

Os índices de risco de Castelli I e || tiveram seus valores médios reduzidos exclusivamente no $\mathrm{GD}$. Em relação à razão $\mathrm{CT} / \mathrm{HDL}-\mathrm{C}$, foi encontrada redução significativa para esse grupo $(P=0,05)$, o que não 
ocorreu com os valores médios da razão LDL/HDL-c, que não alcançou modificações significativas, apesar de apresentar melhora $(P=0,068)$.

Em relação aos TG, não se obteve redução significativa, comparando-se os valores medianos anteriores e posteriores ao programa, em ambos os grupos.

Para todas as variáveis nas quais houve melhora no perfil lipídico, nota-se maior predominância entre os participantes com idade maior que 60 anos, independentemente do grupo.

Não foi encontrada nenhuma correlação entre o sexo dos participantes e as mudanças nos componentes lipídicos.

\section{DISCUSSÃO}

A composição dos grupos foi bastante diferenciada no que diz respeito ao sexo dos participantes, porém tal diferença não interferiu nos resultados, uma vez que nenhuma correlação entre sexo e perfil lipídico foi encontrada.

A idade foi outra variável que diferiu consideravelmente entre os grupos. Indivíduos com idade maior que 60 anos obtiveram maiores efeitos benéficos sobre os componentes lipídicos, embora estes mesmos indivíduos não tenham apresentado redução de peso. Essa constatação pode ser devida à distribuição desigual das idades entre os grupos, já que houve visível predominância de indivíduos idosos no GE, o qual seguiu, em sua média, a mesma tendência em relação ao perfil lipídico e ao peso, independente da idade.

A associação positiva estabelecida entre o IMC e a razão CT/HDL-C, indicou que, quanto mais elevado o IMC, mais alto foi o valor desse indicador. Tal relação ocorreu devido à prevalência de menores níveis de HDL-c estarem associados ao IMC mais elevado, como já foi observado por outro autor ${ }^{(25)}$

Os indivíduos integrantes do GE não apresentaram redução de peso. Tal evidência não exclui, no entanto, uma possível alteração benéfica que o exercício físico possa ter exercido sobre a composição corporal, como o aumento da massa magra ou um não aumento da massa adiposa ${ }^{(26)}$, uma vez que não foram avaliados neste estudo, por exemplo, a circunferência abdominal, a relação cintura/quadril, ou o percentual de gordura corporal.

O GE apresentou redução estatisticamente significativa nos níveis séricos de CT e LDL-C. A redução do LDL-C através da atividade física é consenso entre diversos autores ${ }^{(27-29)}$, no entanto, em relação ao CT, esse benefício ainda é controverso ${ }^{(27,29-30)}$.

Não foi encontrada ação redutora nos níveis de TG ou aumento dos níveis de HDL-c no grupo que praticou exclusivamente exercícios físicos, o que não condiz com estudos semelhantes, que têm mostrado, com poucas exceções, efeitos positivos exercidos pela atividade física sobre esses componentes lipídicos ${ }^{(27-29,31)}$. Alguns autores sugerem que para verificar tais alterações seria necessário um período mais prolongado de estudo. Segundo Superko et al. ${ }^{32)}$, um período de três a nove meses seria suficiente, enquanto para Hartung ${ }^{(33)}$, um prazo de pelo menos seis meses é necessário para obter efeitos mais consistentes.

A literatura indica que o nível inicial dos lipídios séricos influencia os efeitos que a nutrição e a atividade física podem exercer sobre os componentes lipídicos ${ }^{(34)}$. Indivíduos com níveis lipídicos mais elevados, usualmente, possuem maiores decréscimos por mg/dL ${ }^{(34)}$. Assim, podemos pensar que esse tenha sido um fator limitante para obtenção de resultados mais evidentes, já que a análise da amostra, demonstrou valores médios/mediano dentro do recomendado para todas as variáveis correspondentes ao perfil lipídico.

Podemos considerar, ainda, que melhores efeitos sobre o CT, LDLc e TG, além de redução ponderal, poderiam ser obtidos, caso os componentes do GE aliassem, à atividade física, modificações na sua alimentação; afinal, sabe-se que tais variáveis são diretamente influenciadas pela dieta(2,31,35-36)

A falta de controle sobre a prática de exercícios físicos anteriormente ao início do programa também pode ser vista como fator limitante do estudo, considerando que a redução dos níveis séricos dos lipídios tende a sofrer estagnação depois de determinado período de tempo ${ }^{(37)}$

Em relação ao GD, foi possível constatar reduções ponderais, embora elas não tenham sido consideradas estatisticamente significativas. A ausência de maior perda de peso, bem como de efeitos mais consistentes sobre os níveis de CT, LDL-c e TG no GD, opõe-se ao que foi demonstrado por outros estudos $(31,35-36,38)$. Tal fato devese provavelmente à falta de acompanhamento nutricional contínuo durante o estudo; afinal, o retorno às consultas, embora estimulado no primeiro atendimento, na maioria dos casos não foi realizado, seja pelo custo adicional, ou pela falta de planejamento prévio das mesmas.

Apesar de a intervenção nutricional ter sido realizada de forma individualizada e respeitando, sempre que possível, o estilo de vida e os hábitos alimentares dos participantes, o que favorece a adesão à dieta ${ }^{(39)}$, sabe-se que acompanhamento periódico juntamente com a cobrança dos resultados é de extrema importância e, possivelmente, seria um fator determinante para otimização dos resultados obtidos ${ }^{(5)}$. A maior prevalência de obesidade no GD também representa fator dificultante quando se trata de adesão ao tratamento nutricional, pois se verifica, em obesos, maior resistência à modificação de hábitos alimentares já estabelecidos ${ }^{(40)}$.

Os níveis de HDL-c foram elevados apenas entre os componentes do GD, o que acarretou, também, modificações positivas sobre os índices de Castelli I e II; a razão CT/HDL-c apresentou redução estatisticamente significativa. Em relação ao $\mathrm{HDL}$-c e à razão LDLc/HDL-c, ocorreu significância limítrofe, que, provavelmente, seria estatisticamente significativa com o aumento da amostra(41). Vale ressaltar que os integrantes do GD apresentaram, no início do estudo, valores de HDL menores em relação aos integrantes do GE, o que pode ter influenciado melhor resposta nesse grupo, embora diversos outros estudos já tenham demonstrado efeitos benéficos da combinação dieta e exercício físico sobre esse componente lipí$\operatorname{dico}(36,38,42)$.

Sugere-se que o nível de condicionamento aeróbico possua relação diretamente proporcional com os níveis de HDL-c. Devido ao fato de o GD ser composto, predominantemente, por indivíduos obesos (outro viés do estudo), pressupõe-se que os participantes desse grupo obtiveram melhoras mais significativas no seu condicionamento, se comparados com os do GE. Portanto, podemos constatar maior tendência do GD à elevação dos níveis de HDL-c(43)

Embora, em ambos os grupos, não tenha ocorrido redução de peso significativa estatisticamente, os mesmos apresentaram melhoras no perfil lipídico, o que reforça a hipótese de que tanto os efeitos do exercício físico quanto à modificação da dieta acarretam benefícios nos lipídios séricos, independentemente da perda substancial de peso, embora na presença de sinergia entre a perda de peso e a modificação na dieta, esses efeitos sejam mais evidentes ${ }^{(30,44)}$.

Algumas dificuldades foram encontradas na interpretação dos efeitos do exercício físico, bem como do seu efeito combinado com intervenção nutricional, sobre os componentes lipídicos devido a fatores de confusão que interagem com o resultado, tais como: idade, níveis iniciais de lipídios/lipoproteínas, nível de condicionamento aeróbico e redução de peso(45). 
Ao final deste estudo foi possível concluir, que o impacto isolado do exercício físico comparado com seu efeito associado à dieta foi mais evidente em relação as variáveis $\mathrm{CT}$ e $\mathrm{LDL}-\mathrm{c}$. Em relação aos TG, tanto o GE como o GD não demonstraram modificações positivas. No entanto, para as variáveis HDL-c e peso, a associação da dieta com o exercício físico apresentou maiores benefícios.

É importante ressaltar que este estudo possui fatores limitantes, e que esses resultados podem ter sido influenciados pela grande diferença encontrada nos grupos GE e GD. A ausência de pareamento das variáveis idade e sexo entre os grupos também deve ser considerada. O estudo ainda traz outros vieses, como a falta de controle sobre o nível de atividade física anterior ao início do programa, a impossibilidade de determinar os hábitos alimentares dos participantes do GE, além do desconhecimento da adesão ou não dos participantes do $\mathrm{GD}$ à dieta.

Contudo, o presente estudo alerta para a importância da educação nutricional continuada associada à atividade física regular para promover o sucesso do aconselhamento dietético, com controle mais efetivo das dislipidemias e demais fatores de risco cardiovascular.

Assim, são necessários mais estudos que relacionem dieta e exercício físico com os componentes lipídicos, na prevenção e tratamento das dislipidemias.

Todos os autores declararam não haver qualquer potencial conflito de interesses referente a este artigo.

\section{REFERÊNCIAS BIBLIOGRÁFICAS}

1. Garcia RW, Sperandio T, Padezzi J, Lopes G, Alves FR, Duarte TO. Relação entre consumo alimenta atividade física e características antropométricas e os lipídios séricos em pacientes com dislipidemia. Rev Bras Nutr Clin 2002;17:99-106.

2. Santos RD, Maranhão RC, Luz PL, Lima JC, Filho WS, Avezum A, et al. III Diretrizes brasileiras sobre dislipidemias e diretrizes de prevenção da aterosclerose do Departamento de Aterosclerose da Sociedade Brasileira de Cardiologia. Arq Bras Cardiol 2001;77(Suppl 3):1-191.

3. Rique $A B$, Soares $E A$, Meirelles $C M$. Nutrição e exercício na prevenção e controle das doenças cardiovasculares. Rev Bras Med Esporte 2002;8:244-54

4. Organização Mundial da Saúde. Atlas de Doenças Cardíacas e Derrames. Genebra: OMS, 2004

5. Farret JF. Nutrição e doenças cardiovasculares: prevenção primária e secundária. São Paulo: Atheneu, 2005

6. Prado ES, Dantas EH. Efeitos dos exercícios físicos aeróbio e de força nas lipoproteínas HDL, LDL e lipoproteína(a). Arq Bras Cardiol 2002;79:429-33.

7. Isosaki M, Cardoso E. Manual de dietoterapia e avaliação nutricional: Serviço de Nutrição e Dietética do Instituto do Coração-HCFMUSP. São Paulo: Atheneu, 2004.

8. Gus I. Prevenção da cardiopatia isquêmica: o investimento na saúde. Arq Bras Cardiol 1998;70:351-3.

9. American Heart Association. AHA Scientific Statement. Dietary Guidelines. Revision 2000. A Statement for Health Care Professionals from the Nutrition Committee of the American Heart Association. Circulation 2000;102:2284-99.

10. Lottenberg AM. Dietas na hipercolesterolemia. In: Quintão E, editor. Colesterol e aterosclerose. Rio de Janeiro: Qualitymark, 1992;177-94.

11. Grundy SM. Nutrition and diet in the management of hyperlipidemia and atherosclerosis. In: Shils ME, editor. Modern nutrition in health and disease. 9th ed. USA: Williams \& Wilkins, 1998;1199-216.

12. Wright J. Comparación de los efectos de las dietas ricas em hidratos de carbono y las ricas em ácidos grasos mono-insaturados en el control metabolico de pacientes diabéticos e hiperglucémicos. Lecturas Sobre Nutrición 1998:5:60-75.

13. American Heart Association. AHA Scientific Statement. Summary of the Scientific Conference on Dietary Fatty Acids and Cardiovascular Health. Conference Summary from the Nutrition Committee of the American Heart Association. Circulation 2001;103:1034-9.

14. McGowan MP. Am I at risk for developing heart disease? Heart fitness for life. New York: Oxford 1997;10-25.

15. American Heart Association. Summary of Scientific Conference on the Efficacy of Hypocholesterolemic Dietary Interventions. AHA Conference Proceedings. Circulation 1996;94:3388-91.

16. Krumeel D. Nutrição na doença cardiovascular. In: Mahan L, Stump S, editores. Krause: alimentos, nutrição e dietoterapia. 2a. ed. São Paulo: Roca, 1998;543.

17. American College of Sports Medicine. ACM's Guidelines for Exercise Testing and Prescription. 6th ed. Lippincott: Williams e Wilkins, 2000.

18. Ziogas GG, Thomas TR, Harris WS. Exercise training, postprandial hypertriglyceridemia, and LDL subfraction distribution. Med Sci Sports Exerc 1997;29:986-91.

19. Thomas TR, Fontaine T. Exercise and lipoproteins. In: ACSM resource manual for guidelines for exercise testing and prescription. 3th ed. USA: Williams \& Wilkins, 1998;294-301.

20. Blair SN, Horton EH, Fontanez N, Hollerean S, Matheus K, Roheim PS, et al. Physical activity, nutrition, and chronic disease. Med Sci Sports Exerc 1996;28:335-49.

21. Viana FP, Cheik NC, Alexandre SK, Dâmaso AR. Dislipidemias. In: Dâmaso AR, editor. Nutrição e exercício na prevenção de doenças. Rio de Janeiro: Medsi, 2001:200-19.
22. World Health Organization. Preventing and managing the global epidemic: report of a WHO Consultation on Obesity. Geneva: WHO, 1997

23. Castelli WP, Abbot WD, Mc Namara PM. Summary estimates of cholesterol used to predict coronary heart disease. Circulation 1983;67:730-4

24. Callegari J, Sídia M., Bioestatística: princípios básicos e aplicações. Porto Alegre: Artmed, 2003.

25. Lean M. Clinical handbook of weight management. Scotland: Martin Dunitz, 1998

26. Pasman WJ, Sris WHM, Muls E, Vansant G, Westerterp MS. Effects of exercise training on long term weight maintenance in weight-reduced men. Metabolism 1998;48:15-21.

27. MacKnight JM. Exercise considerations in hypertension, obesity, and dyslipidemia. Clin Sports Med 2003;22:101-21.

28. Stfanick ML. Physical activity for preventing and treating obesity-related dyslipoproteinemias. Med Sci Exerc 1999;31(Suppl 11):609-18.

29. Vasankari TJ, Kujala UM, Vasankari TM, Ahotupa M. Reduced oxidized LDL levels after a 10-month exercise program. Med Sci Sports Exerc 1998;30(10):1496-501.

30. Kraus WE, Houmard JA, Duscha BD, Knetzger KJ, Wharton MB, McCartney JS, et al. Effects of the amount and intensity of exercise on plasma lipoproteins. N Engl J Med 2002;347:1483-92.

31. Stone NJ. Focus on lifestyle change and the metabolic syndrome. Endocrinol Metab Clin N Am 2004:33:493-508.

32. Superko HR, Hasked WL, Wood PD. Modification of plasma cholesterol through exercise. Rationale and recommendations. Postgrad Med 1985;78:64-7, 70, 72-5.

33. Hartung GH. High density lipoprotein cholesterol and physical activity: an update: 1983-1991. Sports Med 1993.

34. Hickson JF, Wolinsky I. Nutrição no exercício e no esporte. 2a. ed. São Paulo: Roca, 2002

35. Aldana SG, Greenlaw RL, Diehl HA, Salberg A, Merrill RM, Ohmine S, et al. Effects an intensive diet and physical activity modification program on the health risks of adults. J Am Diet Assoc 2005;105:371-81

36. Verady KA, Jones PJ. Combination diet and exercise interventions for the treatment of dyslipidemia: an effective preliminary strategy to lower cholesterol levels? J Nutr 2005;135:1829-35.

37. Castro I, Schirmer SB, Castro VD, Alcadi R. Fatores de risco coronarianos. In: Batlouni M, Cantarelli E, Ramires JÁ, Luna LR, Feitosa GS, editors. Cardiologia: princípios e prática. Porto Alegre: Artes Médicas Sul, 1999.

38. Sillivan VK. Prevention and treatment of the metabolic syndrome with lifestyle intervention: Where do we start? J Am Diet Assoc 2006;106:668-71.

39. Marchesini SD apud Angelis RC. Riscos e prevenção da obesidade: fundamentos fisiológicos e nutricionais para tratamento. São Paulo: Atheneu, 2003.

40. Sociedade de Cardiologia do Estado de São Paulo. I Diretrizes Nutricionais em Cardiologia. Rev Soc Cardiol Estado de São Paulo 2001:11(Suppl 1):18-57.

41. Altman DG. Practical statitics for medical research. London: Chapman \& Hall, 1991.

42. Rosenson RS. Low HDL-c: a secondary target of dyslipidemia therapy. Am J Med 2005;118:1067-77.

43. Pitanga F, Gondim J. Níveis de prática de atividade física e sua influência sobre o perfil lipídico em indivíduos de ambos os sexos. Rev Bras Ativ Fís Saúde 2000;5:45-53.

44. Betteridge J, Sowers J apud Dâmaso AR. Nutrição e exercício na prevenção de doenças. Rio de Janeiro: Medsi, 2001.

45. Tran ZV, Weltman A, Glass GV, Mood DP. The effects of exercise on blood lipids and lipoproteins: a meta analysis of studies. Med Sci Sports Exerc 1983;15:393-402. 